\title{
Retraction Note to: Key genes associated with osteoporosis revealed by genome wide gene expression analysis
}

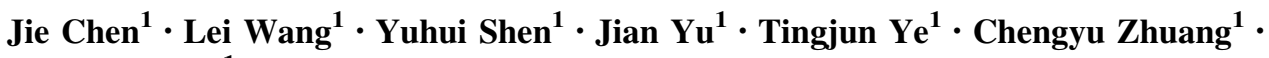
Weibin Zhang ${ }^{1}$

Published online: 18 August 2015

(C) Springer Science+Business Media Dordrecht 2015

\section{Retraction Note to: Mol Biol Rep (2014) 41:5971-5977 \\ DOI 10.1007/s11033-014-3474-1}

The Publisher and Editor retract this article in accordance with the recommendations of the Committee on Publica tion Ethics (COPE). After a thorough investigation we have strong reason to believe that the peer review process was compromised.

The online version of the original article can be found under doi:10.1007/s11033-014-3474-1.

Yuhui Shen

yuhuiss@163.com

$凶$ Weibin Zhang

zhangweiibin@hotmail.com

1 Department of Orthopedics, Shanghai Institute of

Traumatology and Orthopedics, Ruijin Hospital, Shanghai Jiaotong University School of Medicine, 197 Ruijin Er Rd,

Shanghai 200025, People's Republic of China 